
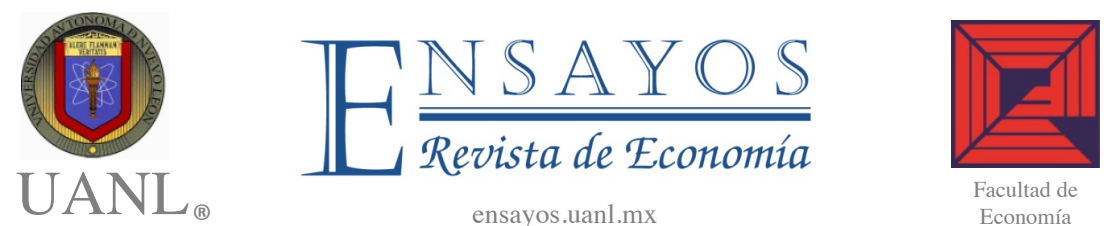

\title{
La apertura comercial de los productos culturales de México (2008-2017)
}

The Trade Openness of Cultural Products of Mexico (2008-2017)

Pablo Sigfrido Corte Cruz*

\begin{tabular}{|c|c|}
\hline $\begin{array}{l}\text { Información del } \\
\text { artículo }\end{array}$ & Resumen \\
\hline $\begin{array}{l}\text { Recibido: } \\
21 \text { junio } 2019\end{array}$ & \multirow{10}{*}{$\begin{array}{l}\text { Este trabajo analiza el grado de participación comercial de } \\
\text { los bienes culturales de México a partir de la Condición } \\
\text { Marshall-Lerner. Se considera un modelo de serie de } \\
\text { tiempo, en el cual se pueda detectar el papel del tipo de } \\
\text { cambio real en la exportación e importación de los } \\
\text { productos culturales, a partir de los datos del Sistema de } \\
\text { Cuentas Satelitales de Cultura, que publica el Instituto } \\
\text { Nacional de Estadística y Geografía (INEGI). Los } \\
\text { resultados señalan que la balanza comercial de cultura es } \\
\text { inelástica; esto implica la demanda interna de productos } \\
\text { similares provenientes del exterior, principalmente, de } \\
\text { Estados Unidos. Si bien hay un alto grado de apertura } \\
\text { comercial, esto no se refleja en el sector de la cultura. Se } \\
\text { concluye que, a pesar de la apertura comercial, la } \\
\text { influencia cultural de México es mínima. }\end{array}$} \\
\hline $\begin{array}{l}\text { Aceptado: } \\
22 \text { abril } 2020\end{array}$ & \\
\hline Clasificación JEL: & \\
\hline C22; F14; F31; Z11; & \\
\hline Z13; Z19 & \\
\hline Palabras clave: & \\
\hline Balanza Comercial de & \\
\hline la Cultura; Condición & \\
\hline Marshall-Lerner; & \\
\hline Bienes Culturales & \\
\hline
\end{tabular}

\footnotetext{
*Benemérita Universidad Autónoma de Puebla, pabloscc71@gmail.com
}

ISSN Electrónico: 2448-8402 | ISSN Impreso: 1870-221X | (C2020 Los autores @) () 


\begin{tabular}{|c|c|}
\hline Article information & Abstract \\
\hline $\begin{array}{l}\text { Accepted: } \\
22 \text { April } 2020\end{array}$ & $\begin{array}{l}\text { This paper analyzes the degree of commercial } \\
\text { participation of the cultural goods of Mexico from the } \\
\text { Marshall-Lerner's Condition. I use a model of time series, } \\
\text { in which the role of the real exchange rate in the export } \\
\text { and import of cultural products can be detected, based on }\end{array}$ \\
\hline $\begin{array}{l}\text { JEL Classification: } \\
\text { C22; F14; F31; Z11; } \\
\text { Z13; Z19 } \\
\text { Keywords: } \\
\text { Trade Balance of } \\
\text { Culture; Marshall- } \\
\text { Lerner's Condition; } \\
\text { Culture Goods }\end{array}$ & $\begin{array}{l}\text { data from the System of Cultural Satellite Accounts, } \\
\text { published by the Instituto Nacional de Estadística y } \\
\text { Geografia (INEGI). The results indicate that the trade } \\
\text { balance of culture is inelastic; this implies the role of } \\
\text { domestic demand for similar products from abroad, mainly } \\
\text { from the United States. While there is a high degree of } \\
\text { trade openness, this is not reflected in the culture sector. In } \\
\text { conclusion, despite the trade opening, the cultural } \\
\text { influence of Mexico is minimal. }\end{array}$ \\
\hline
\end{tabular}

\section{Introducción}

Si bien la cultura se ha considerado como un bien no comerciable, la publicación de los Sistemas de Cuentas Satelitales sobre este sector, inciden en analizar su papel en la economía, específicamente en lo que se refiere a los aspectos del espectáculo, además de las actividades artísticas. A partir de esto, queda claro que el concepto cultura, dentro de la economía, va más allá de la explicación de sociólogos y antropólogos que se han limitado, por lo regular, a explicar los usos y costumbres al interior de los procesos de intercambio económico.

Para el caso de México, de acuerdo con el Instituto Nacional de Estadística y Geografía (INEGI, 2018), la cultura representa 3.2\% del total del Producto Interno Bruto (PIB) de 2017, lo cual es similar a la aportación del total del Sector Primario, es decir, tiene mayor importancia que la misma agricultura, en el total de la economía.

La cultura, en términos de empleo, ha sido de lo más representativo en la última década, en 2017, 3.2\% del total del empleo se concentra en este sector (INEGI, op. cit.)

El estudio de este sector es cada vez más atractivo, no solo para los estudiosos de las llamadas ciencias humanas, sino también para los economistas, al considerar los datos publicados tanto por el INEGI como los del Sistema de Información Cultural (SIC), que difunde la Secretaría de Cultura Federal.

En nivel mundial, el análisis económico de la cultura no es nuevo. En el año 1966, William Baumol y William Bowen publicaron el libro Performing Arts: The Economic Dilemma, donde se revisan los costos y financiamientos 
públicos y privados de las artes, así como sus retribuciones; para el caso mexicano resulta novedoso con un creciente número de economistas que han iniciado su estudio.

Con las diversas publicaciones electrónicas de información estadística, que realiza el INEGI, se puede especificar el papel que juega la cultura, tanto en la demanda interna como en la conformación del valor agregado de la misma. Del mismo modo, se establece que, al igual que otros sectores de la economía, la cultura también implica relaciones comerciales.

El objetivo de este documento es realizar un ensayo sobre las relaciones comerciales de la cultura, a través de la condición Marshall-Lerner, lo cual conlleva su relación con el Tipo de Cambio Real sobre las exportaciones e importaciones de este sector.

Si bien algunos artistas mexicanos han obtenido renombre internacional, ya sea por su trabajo o por su acercamiento a los medios de comunicación, la influencia cultural de México es mínima, siendo esta la hipótesis a sostener.

Para reafirmar la hipótesis anterior, se analizan tres aspectos desde una revisión teórica, como: el tamaño de la economía, las preferencias de consumo y el nivel de reconocimiento del artista o compañía en términos de la demanda externa.

Para la demostración, se revisa, además del Producto Interno Bruto (PIB) de la cultura, a subsectores como las Artes Visuales y Plásticas (dominados por la fotografía y el comercio de artes plásticas), Música y Conciertos (música, bienes comerciales para músicos y conciertos) y Medios Audiovisuales (Cine, Radio, Televisión, Internet, entre otros), los cuales han mostrado una importancia considerable en términos de la balanza comercial de la cultura.

Se realiza, como ya se ha señalado, una revisión teórica que ayude a explicar la importancia de la relación entre cultura y comercio internacional, especialmente en lo que se refiere a los bienes y servicios culturales, misma que se establece en la siguiente sección. Posteriormente, se formula el modelo econométrico, a partir de lo expuesto en diferentes documentos relacionados con la Condición Marshall-Lerner. Los resultados, discusión y reflexiones finales se presentan en las secciones subsiguientes.

\section{Revisión teórica}

Para analizar la literatura sobre el comercio internacional de los bienes culturales, se tienen que identificar tres aspectos: 1) El tamaño de la economía, 2) las preferencias de consumo de acuerdo con la cercanía de los productos extranjeros, en comparación con los nacionales de características similares y 
3) el grado de influencia de un bien o servicio cultural, a partir de la marca comercial establecida o reconocida en dicha región o país.

Esta revisión tiene como propósito verificar las opiniones de algunos autores dedicados al estudio de la Economía de la Cultura, en referencia a cada uno de los aspectos ya mencionados, con la finalidad de afirmar el grado de influencia de las economías pequeñas en el comercio internacional, en lo que corresponde a los bienes culturales transables.

\subsection{Comercio de bienes culturales y tamaño de la economía}

Para el estudio del primer aspecto, no solo se considera el tamaño de la economía, sino también características como los usos y costumbres, la historia, la política, el lenguaje, entre otros, que son de transmisión familiar y social; pero se destaca que, todos estos, implican costos en los procesos de negociación.

De acuerdo con István Kónya (2006), el tamaño de la economía influye en las condiciones del comercio internacional, debido a la existencia de diferencias culturales que imponen ciertas barreras a la apertura comercial, lo cual se traduce en costos.

Las diferencias culturales, según este autor, no deben implicar un obstáculo, sino que al igual que la tecnología, los aspectos idiosincráticos se superan por completo:

Si bien los costos tangibles del comercio también pueden reducirse mediante la inversión, en cualquier momento esto está limitado por el estado de la tecnología. Por otro lado, las barreras culturales pueden superarse por completo, por lo que el aprendizaje no está limitado por factores tecnológicos sino por factores de mercado. (Kónya, op. cit.: 495).

Por tanto, estas diferencias se superan a medida que se realiza una inversión suficiente y redituable, que permita el pleno entendimiento de las negociaciones comerciales entre dos economías diferentes, incluso con idiomas diferentes.

De hecho, el mismo Kónya (op. cit.) establece que la principal barrera cultural es el idioma, pero esto no obliga a que ambas partes aprendan el lenguaje de su contraparte, por ejemplo, si Estados Unidos y México se encuentran en relaciones comerciales, los estadounidenses no requerirían hablar español si los mexicanos invierten en hablar en inglés. 
En otros términos, las economías pequeñas invierten más en aprender de la cultura de la economía grande, pero no al revés, a menos que la primera subsidie el aprendizaje en el segundo país, siempre y cuando, esto sea retribuido en términos comerciales:

Los principales resultados ... se resumen a continuación: (i) los países pequeños invierten relativamente más en aprendizaje, mientras que las naciones grandes pueden no tener ningún aprendizaje; (ii) los equilibrios múltiples son posibles cuando los dos países son de tamaño similar; (iii) el efecto de la globalización en el aprendizaje es ambiguo y depende fundamentalmente de si los costos físicos o culturales del comercio están disminuyendo; (iv) se presenta una caída cada vez mayor de los costos tangibles del comercio, previa a la disminución de la apertura cultural de los grandes paises; (v) la cantidad de aprendizaje en equilibrio siempre es inferior al óptimo en general, pero es posible que los paises individuales inviertan demasiado; y (vi) subsidiar el aprendizaje en el extranjero puede mejorar el bienestar de ambos países, si el país imponente es relativamente pequeño (Kónya, op. cit.: 504).

Tania Voon (2008) afirma que el comercio internacional contrae un dominio de aspectos culturales de las economías grandes sobre las pequeñas. En este caso, las decisiones de consumo de las economías pequeñas se basan en lo proveniente de las economías grandes, incluyendo procesos de aprendizaje del país dominante.

Esto último, contrae que algunos países, con presencia de un alto patrimonio histórico, impongan una especie de 'proteccionismo cultural', por lo cual, Voon (op. cit.) propone que se establezcan legislaturas de comercio internacional para estos términos; sin embargo, de acuerdo con la autora, para desarrollar esto se debe obtener un concepto de cultura que implique su universalidad y su diversidad.

Se está más allá de... establecer una definición definitiva y resumida de cultura, o incluso una definición de cultura para los fines del derecho mercantil internacional. La cultura es un concepto disperso y sin forma. En el contexto del derecho internacional, a pesar de la proliferación de tratados y declaraciones sobre el tema, no existe una definición acordada de cultura. Sin embargo, como ejemplo del significado potencialmente expansivo de la cultura, el preámbulo de la Declaración Universal sobre la Diversidad Cultural define la cultura como 'el conjunto de características distintivas espirituales, materiales, intelectuales y emocionales de la sociedad o de un grupo social, [que abarca], incluso, el arte y la literatura, estilos de vida, formas de convivencia, sistemas de valores, tradiciones y creencias' (Voon, op. cit.: 12). 
La cultura puede traducirse tanto en el entorno de los usos, costumbres y protección del patrimonio; pero, también puede entenderse como expresión de las diversas artes que se consideran universales.

Así mismo, ante los procesos de apertura comercial y, con la llegada de productos que perjudican algunos aspectos culturales -como los hábitos de calzado, vestimenta, educación, religión, procesos de producción agrícola y ganadera, entre otros-, en algunos países, se establecen legislaciones para proteger las conductas tradicionales, sin afectar las relaciones comerciales.

Bisin y Verdier (2014) analizan el papel de la diversidad cultural en las relaciones comerciales. Señalan que, si bien el comercio internacional motiva el desarrollo económico, por otro lado, puede afectar a la diversidad cultural de la región, más aun, donde se localizan idiomas étnicos correspondientes a la zona.

Más allá de los estudios de caso, la literatura empírica sobre comercio, globalización, integración económica y cultura aún no es concluyente. Por un lado, la idea de que el comercio y la globalización se asocian con una reducción de la diversidad cultural es consistente con algunas pruebas que sugieren la convergencia de los patrones de consumo en todos los países (Bisin y Verdier, op. cit.: 441).

En ese sentido, las relaciones comerciales implican procesos de integración cultural, lo cual contrae costos de coordinación, por ejemplo, el aprendizaje de un idioma. Si existe un solo individuo o un grupo pequeño de individuos, este proceso de integración será sencillo en comparación si el grupo es demasiado grande .

Bisin y Verdier (op. cit.) señalan que los procesos de integración cultural tienen incentivos meramente económicos, como lo son los beneficios y apoyos sociales de grupos vulnerables, los que pueden ser motivados a mezclarse con el grupo mayoritario.

Los efectos de los procesos de apertura comercial en la integración cultural pueden ser ambiguos, a decir:

... a corto plazo, la globalización proporciona beneficios de utilidad a partir de una mayor interactividad social, la competencia internacional con fuertes efectos en el tamaño del mercado puede llevar a una menor diversidad cultural, menos creatividad cultural y una menor calidad de los bienes culturales en el futuro (Bisin y Verdier, op. cit.: 447). 
Por otro lado, la integración comercial no solo motiva el intercambio de bienes, sino también el de factores, lo cual reduce los costos de producción por ambas partes, y genera convergencias culturales, tanto en el nivel individual como institucional, motivando la reducción de gastos, además del pleno entendimiento de la diversidad de regiones (Bisin y Verdier, op. cit.).

Ante el estudio de estos tres textos, aquí expuestos, se puede deducir que las economías grandes estarían dispuestas a gastar en procesos de integración cultural, sí solo sí, pequeños grupos buscan adoptar las costumbres del grupo mayoritario, de lo contrario no se estaría en dicha disposición. Por otro lado, la economía pequeña gastaría en procesos de aprendizaje en conocer la cultura de la economía grande, pero no necesariamente ocurre lo contrario.

Por tanto, las economías pequeñas estarían dispuestas a invertir para mejorar las relaciones comerciales con las economías grandes, pero ante esto, se deben considerar las condiciones de patrimonio, usos y costumbres, así como los procesos de consumo y producción de ambas partes. Esto último puede conllevar costos quizás más altos que los beneficios posibles obtenidos.

Esta idea, puede ser trasladada en términos de bienes y servicios culturales, considerando como ejemplo la presencia de un laudero de Michoacán, que se dedica a la producción de Guitarras de Concierto. En este caso, su venta al exterior es al por menor, y en la mayoría de estos casos, es una producción sobre pedido. No se niega la alta calidad de un instrumento musical de este tipo de hechura artesanal en nuestro país, pero, por otro lado, una empresa estadounidense, fabricante de guitarras eléctricas, debido a que su producción es masiva, implica costos cada vez más accesibles a las tiendas de música de cualquier parte de México.

\subsection{Las preferencias de consumo}

Las preferencias de consumo son el resultado del tamaño de la economía. Un ejemplo es el de la industria cinematográfica de Hollywood en comparación con la producción filmográfica mexicana. En la primera, debe tomarse en cuenta que existen grandes inversiones, por lo regular del sector privado, que cuenta con llamativos efectos especiales, mismos que le permiten ofrecer un buen producto al consumidor. En el caso del cine mexicano, cuyo presupuesto (por lo regular escaso) proviene principalmente del sector gubernamental y no contiene la espectacularidad de su referente estadounidense, no resulta ser atractivo al consumo popular.

Además, en términos de cantidad, la producción de cine norteamericano es muy superior a la del caso mexicano. Bajo esta consideración, la adquisición 
de entradas para ver la filmografía estadounidense, en México, es mucho más elevada en comparación con la nacional.

En este caso, James E. Rauch y Vitor Trindade (2009), señalan que esto no es nuevo, pues a partir de la Segunda Guerra Mundial, la influencia de la cultura estadounidense se refleja en el nivel mundial, sobre todo en la generación de los jóvenes de los años cincuenta y sesenta, con el desarrollo de la industria musical. Pero, con la expansión del libre comercio, aunado a la reducción de costos en las comunicaciones, la presencia del internet y otros medios que han fomentado nuevas interacciones sociales, se ha incrementado el mercado cultural de Estados Unidos.

Así como se presenta en la industria cinematográfica, este suceso también ocurre en música, el baile, las artes plásticas, entre otras manifestaciones artístico-culturales, con lo que se muestra que las economías pequeñas consumen la cultura proveniente de las economías grandes. Este efecto se intensifica a partir de la presencia de nuevos y desarrollados medios de comunicación, como los smartphones, televisión de paga, redes sociales, entre otros, por lo que:

Si todos se comunican por igual con todos los demás, el estándar del país más grande tenderá a tomar el control, mientras que existe una mayor posibilidad de que la producción "nicho" sobreviva, si los grupos están aislados de la comunicación entre ellos, y tienen diferentes preferencias subyacentes (Rauch y Trindade, op. cit.: 810).

Entonces, al considerar los procesos de la vida moderna actual, la extensión de ideas y bienes culturales influyen en futuros procesos culturales (Rauch y Trindade, op. cit.). Y aunque esto no es nuevo, siempre se considera que la influencia de la cultura extranjera puede afectar los saberes domésticos.

Estos procesos de comunicación generan un consumo de bienes culturales provenientes del exterior, tanto por calidad como por cantidad. Si bien hay bienes culturales domésticos similares, estos no llegan a ser sustitutos perfectos sino que más bien, llegan a ser sustitutos imperfectos (Rauch y Trindade, op. cit.).

Según estos autores, primero se debe desarrollar el consumo interno de los bienes culturales domésticos, generando una red de externalidades, mismas que se pueden promover posteriormente al exterior.

Las externalidades de la red de consumo refuerzan el efecto en el mercado interno, siendo el refuerzo más fuerte para los bienes culturales, para los cuales las externalidades de la red de consumo son más fuertes. El mayor 
intercambio de las externalidades de la red de consumo, entre los países, también exacerba el efecto del mercado interno, por lo que el aumento de la comunicación y los viajes entre países aumentarán la participación en el mercado cultural del país más grande (Rauch y Trindade, op. cit.: 833).

Ante este tipo de efectos de consumo de bienes culturales, Voon (op. cit.) pone algunos ejemplos de políticas de "protección" de cultura por parte de algunos países miembros de la Organización Mundial de Comercio (OMC). De los mencionados por la autora, el caso canadiense es el más emblemático, pues en su legislación sobre radiodifusión, se establecen reglas de porcentaje mínimos y máximos de transmisión, que muestran la creación y presentación de bienes culturales domésticos. Este tipo de legislaciones ha sido ejemplo a seguir para otros miembros de la OMC. Para la investigadora de la Universidad de Melbourne, estos procesos dependen del papel que juegan las "industrias culturales", puesto que algunos bienes culturales, pueden ser comerciables.

Aunque la industria cultural es un negocio como cualquier otro, los productos culturales tienen características culturales, no comerciales, que los distinguen de otros bienes y servicios comerciables. Y las ventas de productos culturales locales en el mercado pueden no reflejar adecuadamente el valor cultural de esos productos para la comunidad en general (Voon, op. cit.: 33).

Sin embargo, el tema de la cultura queda a un lado en las negociaciones comerciales (Voon, op. cit.), lo que ha permitido una mayor expansión de la cultura estadounidense, ante la ausencia de "industrias culturales" en las economías pequeñas.

\subsection{El grado de influencia de los bienes y servicios culturales}

Aunado con el tipo de preferencias de consumo en bienes culturales, se destaca el posicionamiento de algunos "productos" por su reconocimiento y calidad en cualquier parte del mundo, lo cual lleva a una discusión, si el sector cultural debe estar, o no estar, protegido. En ese sentido, Ágúst Einarsson (2016) señala:

Hay argumentos a favor y en contra del tratamiento especial de los bienes culturales. Entre los argumentos a favor se encuentra el argumento de que los bienes culturales tienen un valor que trasciende el mero valor económico y que los mercados tradicionales no reflejan adecuadamente ese valor. Además, los bienes culturales son fundamentales para la autopercepción y las identidades nacionales de cada país y, por lo tanto, deben protegerse para el beneficio público. Una fuerte producción cultural nacional es importante para la diversidad cultural, que podría verse amenazada por importaciones baratas, que a menudo disfrutan de sus ventajas de precio para combatir la 
competencia, y por esta razón se debe mantener una estrecha vigilancia sobre el comercio mundial de bienes culturales para evitar una ventaja injusta, una que no está orientada al mercado. Finalmente, la industria cultural a menudo es débil, especialmente al principio y, por lo tanto, debe gozar de cierta protección en su fase de desarrollo. También hay algunos argumentos en contra del tratamiento especial para los bienes culturales, el principal argumento es que la protección especial resultará en una distorsión del mercado y conducirá a la ineficiencia. También se menciona con frecuencia que el impulso de protección es muy tendencioso y se origina entre los participantes del mercado que buscan protegerse a sí mismos y a sus propios intereses, en lugar de a sus respectivas culturas. Finalmente, se argumenta que la protección implica restricciones a la libertad individual de elección, y que el libre comercio de bienes culturales, aumenta la diversidad cultural ( $\mathrm{p}$. 116-117).

Es obvio que, dicha discusión impone normativas hacia un concepto amplio de cultura, por encima de las condiciones de patrimonio, turismo, usos y costumbres, entre otros, aplicando las condiciones propias de consumo popular y de medios.

Es decir, el intercambio de bienes y servicios culturales a través del comercio otorga los mismos fenómenos comerciales de exportación e importación, ya que existen procesos de demanda interna versus oferta externa y viceversa, que generan aspectos propios de una balanza comercial cultural, con sus propios superávits y déficits, según la posición comercial que se establezca.

El hecho de que exista un consumo por bienes culturales externos, implica una producción de los mismos por parte de industrias culturales, lo cual, contiene una marca que le permite ser comercializado.

En la mayoría de los casos, la marca se debe a las condiciones de mercado existentes, tal es el caso de las industrias: cinematográfica, discográfica y de la impresión, las que establecen sus premiaciones a lo más destacado, o a lo más vendido del sector.

Rauch y Trindade (op. cit.) establecen esta importancia, ofreciendo como ejemplo la competencia de las películas de Estados Unidos en los mercados europeos y asiáticos, versus las creaciones domésticas.

Continuando con el ejemplo de la industria cinematográfica, en México se localizan, básicamente, empresas como Canana Films, Bandidos Films y Ánima Estudios, entre otras realizadoras que son subsidiadas por las cadenas de televisión abierta. Las cuales son consideradas debido a la exportación de sus películas; sin embargo, en el mercado doméstico, tienen que competir con 
las grandes empresas estadounidenses como Disney, Universal Studios, Dreamworks, Paramount, entre otras tantas miles que se establecen como una marca reconocida.

Otro ejemplo es la industria de la música popular, en la cual, las empresas discográficas dominantes en México, en su mayoría, son sucursales de las provenientes de Estados Unidos. Con respecto al entretenimiento, principalmente la creación de dibujos animados, los artistas mexicanos se trasladan a estudios de Estados Unidos para desarrollar sus habilidades, o se subcontratan con empresas productoras de dicho país, fortaleciendo la presencia de las marcas estadounidenses en el ramo.

También la marca puede corresponder al concepto del prestigio de un artista o grupo artístico, lo cual ya significa un valor agregado a la venta de sus productos culturales. Se puede pensar en cineastas, tanto mexicanos como estadounidenses, compitiendo por la taquilla en ambos lados de la frontera. Indudablemente en ambos mercados, los últimos superan a los primeros, puesto que, a pesar de que ser todos reconocidos, el dominio anglosajón es generador de altas ganancias.

Igual ocurre con las compañías artísticas de Europa y Estados Unidos que, debido a su prestigio, pueden considerarse como marcas comerciales; la presentación de cualquiera de ellos en foros y auditorios de cualquier parte del mundo, implica un lleno total. En México, a pesar de existir instituciones reconocidas internacionalmente, no se asegura un aforo similar.

Una marca refleja la confianza entre una empresa y sus clientes y, por lo tanto, es importante para la marca reflejar la imagen de la empresa y su vínculo positivo con los clientes. También es importante que la marca contribuya a la creación de una base de clientes leales y estables donde los clientes se involucren en un negocio estable, por ejemplo, asistiendo a actuaciones regulares en un teatro determinado o comprando y leyendo todos los libros de un novelista en particular. Las empresas comerciales, por lo tanto, tienen que cuidar bien de sus marcas. En el sector cultural, a menudo es de primordial importancia para las experiencias de los clientes cumplir con las expectativas planteadas por una marca, ya sea que la marca esté asociada a un artista individual, una orquesta, una editorial o cualquier otra entidad que produzca bienes culturales (Einarsson, op. cit.: 123).

El prestigio genera una marca para la venta de productos culturales, pero al reconsiderar el primer punto sobre el tamaño de la economía, es evidente que las compañías artísticas de Estados Unidos y Europa dominan en nivel mundial, por lo que los intercambios culturales pueden ser desiguales, lo 
mismo que las condiciones de exposición de expresiones culturales con respecto a las economías pequeñas.

Los intercambios culturales entre países pueden ser extensos, incluso si no implican una actividad económica significativa. Estos intercambios pueden tomar la forma de mercados con artistas que trabajan a través de agentes o agencias, como en el caso de los cantantes de ópera que viajan con las compañias de teatro de ópera y se desempeñan en los roles que han elegido dominar. Los contratos de este tipo a menudo se hacen con muchos años de anticipación. Los artistas siempre han viajado de esta manera de país en país, las compañías de teatro y orquestas son ejemplos, y algo similar sucede en el caso de las películas, que a menudo se producen en cooperación entre dos o más países (Einarsson, op. cit.: 118).

Regresando al ejemplo de la industria musical, no es lo mismo un concierto de un grupo musical de alto reconocimiento mundial, en comparación con un conjunto de música tradicional, regional (incluso pop), que se establezca en los mercados internacionales .

En conclusión, los tres elementos aquí destacados implican que las condiciones de intercambio comercial de la cultura son desiguales, pues se promueve el mercado de las producciones extranjeras, mientras que la capacidad de exportación nacional no es mucho mayor, a pesar del prestigio ganado por creadores mexicanos en los mercados mundiales.

\section{Modelo}

\subsection{El grado de apertura comercial}

Para demostrar que la demanda de cultura en México es mínima, en comparación con otros sectores en el mercado internacional, primero, se debe establecer el indicador básico sobre el Grado de Apertura Comercial (GAC) de nuestro país, el cual sirve para medir la evolución del comercio. De acuerdo con la Organización para la Cooperación y Desarrollo Económicos (OCDE, 2010), el GAC es el flujo de comercio con respecto al total de la economía, medido por su Producto Interno Bruto (PIB).

$G A C=\frac{X+M}{P I B} \times 100$

Rafael López Del Paso (2014) señala que este tipo de mediciones sirve para medir el GAC de las economías pequeñas, debido a que son más especializadas; mientras que las economías grandes, "dedican una mayor proporción de su producción a su mercado interior” (p. 87). 
Además de considerar la apertura total de la economía, se realizan las operaciones correspondientes a tres subsectores que resultan representativos, en estos términos, los cuales son las Artes Visuales y Plásticas, Medios Audiovisuales y Música y Conciertos.

\subsection{Condición Marshall-Lerner}

Este trabajo se propone analizar si existe un efecto positivo ante la depreciación del tipo de cambio real en la Balanza Comercial de la Cultura. Para esto, se requiere de la llamada Condición Marshall-Lerner.

De acuerdo con Francisco Rivera-Batiz y Luis A. Rivera-Batiz (1993), la balanza comercial se mide a partir del valor de las exportaciones con respecto al de las importaciones, medidas en término del precio relativo de los bienes domésticos, esto es:

$T=M^{*}\left(q, Y^{*}\right)-q M(q, Y)$

donde $\mathrm{T}$ es la balanza comercial, $M^{*}$ es la demanda externa por bienes domésticos, es decir, las exportaciones que están en función del Tipo de Cambio Real $(q)$ y el nivel de ingreso del exterior $\left(Y^{*}\right)$, y $M$, que se define como la demanda interna por bienes externos; en otro sentido, las importaciones que también dependen del Tipo de Cambio Real, pero además del nivel de ingresos interno $(Y)$.

De acuerdo con esto, un incremento en $Y^{*}$ mejora las exportaciones, mientras que un aumento en los ingresos internos implica una mayor compra de bienes externos. En relación con q, una depreciación implicaría precios más competitivos para los bienes internos en el exterior, y un encarecimiento de los bienes extranjeros.

Si se mantienen constantes los dos niveles de ingreso, el cambio en la balanza comercial, dado por (2), se mide como:

$\Delta T=\Delta M^{*}-q \Delta M-\Delta q M$

del cual, al ser medido con respecto a las variaciones en el Tipo de Cambio Real, se tendría,

$\frac{\Delta T}{\Delta q}=\frac{\Delta M^{*}}{\Delta q}-q \frac{\Delta M}{\Delta q}-M \frac{\Delta q}{\Delta q}$ 
Si se partiera del supuesto de que la $T$ se encontrara en equilibrio $\left(M^{*}=q M\right)$, entonces,

$$
\frac{\Delta T}{\Delta q}=\frac{M^{*}}{q}\left(\frac{\Delta M^{*}}{\Delta q} \frac{q}{M^{*}}-\frac{\Delta M}{\Delta q} \frac{q}{M}-1\right)
$$

el cual puede reescribirse como:

$$
\frac{\Delta T}{\Delta q}=\frac{M^{*}}{q}\left(\eta^{*}-\eta-1\right)
$$

donde $\eta^{*}=\frac{\Delta M^{*}}{\Delta q} \frac{q}{M^{*}} \mathrm{y} \eta=\frac{\Delta M}{\Delta q} \frac{q}{M}$, que representan las elasticidades precio de la demanda de exportaciones e importaciones, respectivamente.

Por tanto, la entidad (2d) establece la llamada condición Marshall-Lerner, donde la suma de los valores absolutos de las elasticidades precio de las exportaciones e importaciones, debe ser superior a la unidad.

$$
|\eta *|+|\eta| \geq 1
$$

Al cumplirse dicha condición, una depreciación mejora la balanza comercial a favor de nuestros bienes, en caso contrario, no ocurriría así .

\subsection{La literatura sobre modelos para analizar la condición Marshall- Lerner}

Un análisis requiere series de tiempo y que se revisen diferentes propuestas realizadas por algunos especialistas en Comercio Internacional. Entre las que sobresalen los modelos VAR (Vector Autorregresivo) y, en su caso, modelos VEC (Vector de Corrección de Error), para demostrar la condición MarshallLerner, debido a que están diseñados para analizar los efectos de elasticidades para el largo plazo.

Entre las razones de utilizar este tipo de Modelos Autorregresivos de Rezagos Distribuidos (ADL), se argumenta que: 1) se requiere de modelos dinámicos para analizar el comportamiento de las elasticidades que se estudian y, 2) las variables no son estacionarias.

Para la aplicación de este tipo de modelos, se requiere de algún grado de diferenciación para saber si existe, o no, la cointegración, pues al aplicar un 
modelo de Mínimos Cuadrados Ordinarios (MCO), se violan supuestos básicos (Ramírez, Azuara, Calderón y Candelaria, 2008; Bustamante y Morales, 2009).

Este tipo de modelos ADL tienen un buen fundamento para ser utilizados, porque se considera que hay elementos de la misma variable dependiente, que se puede explicar a sí misma, en términos de sus propios rezagos, y por otro lado, se busca corregir la autocorrelación de los errores.

\subsection{Los modelos ARCH-GARCH}

Si bien, los modelos VAR-VEC son buenas propuestas de análisis para la condición Marshall-Lerner, existen algunas críticas sobre problemas como la presencia de la heterocedasticidad, la cual no se corrige del todo.

De ahí la importancia de utilizar modelos ARCH-GARCH (Autorregresivos Considerando la presencia de Heterocedasticidad y ARCH Generalizado), tal y como lo proponen Baltazar y Ramos (2014), para medir la volatilidad de las variables que se analizan en este trabajo. El modelo econométrico a desarrollar se especifica de la forma:

$\Delta \log M^{*}=$

$$
\begin{aligned}
\beta_{0}+\beta_{1} \Delta \log q+ & \beta_{2} \Delta \log Q^{*}+\Sigma \Phi_{i} \Delta \log M^{*}{ }_{\mathrm{t}-\mathrm{i}} \\
& +\Sigma \Theta_{i} u_{\mathrm{t}-\mathrm{i}}+\Sigma \gamma_{i} \varepsilon_{\mathrm{t}-\mathrm{i}}^{2}+\Sigma \Omega_{i} \sigma_{\mathrm{t}-\mathrm{i}}^{2}
\end{aligned}
$$

$\Delta \log M=$

$$
\begin{aligned}
\beta_{0}+\beta_{1} \Delta \log q+ & \beta_{2} \Delta \log Q+\Sigma \Phi_{i} \Delta \log M_{\mathrm{t}-\mathrm{i}} \\
& +\Sigma \Theta_{i} u_{\mathrm{t}-\mathrm{i}}+\Sigma \gamma_{i} \varepsilon_{\mathrm{t}-\mathrm{i}}+\Sigma \Omega_{i} \sigma_{\varepsilon}^{2}{ }_{\mathrm{t}-\mathrm{i}}
\end{aligned}
$$

En este caso, las variables se expresan en términos de logaritmos naturales de las exportaciones $\left(M^{*}\right)$, las importaciones $(M)$ y el Tipo de Cambio Real $(q)$. De igual forma, $Q^{*}$ y $Q$ son los índices de PIB de Estados Unidos y de México, respectivamente, consideradas como variables proxys para medir el ingreso. Los parámetros $\Phi_{\mathrm{i}}$ representan a los procesos autorregresivos (AR) de las variables dependientes correspondientes, mientras que $\Theta_{i}$ a los procesos de Media Móvil (MA). Así mismo, los parámetros $\gamma_{i}$ y $\Omega_{i}$ explican los procesos ARCH y GARCH, respectivamente.

Los parámetros $\beta_{1}$, tanto en (4) como en (4a) son las elasticidades-precio de las exportaciones e importaciones, respectivamente; la suma absoluta de ambos debiera cumplir la condición Marshall-Lerner para los efectos de la economía de la cultura. 


\section{Fuente y tratamiento de los datos}

La información correspondiente al PIB de la Cultura, así como de los subsectores de estudio, se obtienen del Sistema de Cuentas Satelitales de la Cultura (CSC) que publica el INEGI en su página electrónica. Al ser información de precios nominales, se tienen que deflactar, teniendo como base al Índice Nacional de Precios del Consumidor que publica el Banco de Información Económica (BIE-INEGI), esta última fuente también es recurrida para la información del PIB.

La información correspondiente al Tipo de Cambio y el Índice Nacional de Precios al Consumidor, de igual forma, se localizan en el BIE-INEGI.

Con respecto al PIB de Estados Unidos y su índice, al igual que su índice de precios, estas se localizan en la página electrónica del Bureau of Economic Analysis (BEA, sf).

La aplicación de un modelo del tipo ARCH-GARCH implica que la información recabada tenga cierto grado de frecuencia para una mayor eficiencia en la obtención del resultado de los coeficientes. Por dicha razón, los datos extraídos de las fuentes de información mencionadas, tienen que ser transformados en mensuales, debido a que la información sobre tipo de cambio e índices de precios tiene dicha frecuencia.

El primer dato que se transforma es el correspondiente al PIB, cuya frecuencia es trimestral. Con respecto a esto, se utiliza el llamado Filtro de Kalman, el cual, según Rocío Elizondo (2012), es el más robusto de los tratamientos debido a que requiere de una variable cercana con uno o dos meses de rezago, para construir los datos no observables que se solicitan.

Para el caso del PIB, la variable más cercana es el Indicador Global de Actividad Económica (IGAE). Para la transformación de información trimestral a mensual se requieren de dos pasos:

... el primero corresponde a estimar los parámetros con datos trimestrales observados del PIB y del IGAE y posteriormente usar el filtro de Kalman con los parámetros ya conocidos para aproximar el PIB mensual, a este procedimiento se le llama método de una etapa (M una Etapa); el segundo se basa en estimar conjuntamente los parámetros y el PIB mensual, a este método se le llama método de filtro de Kalman (MFK) (Elizondo, op. cit.: 4).

Para el caso de la economía estadounidense, la conversión de su PIB se realiza a partir de su índice de producción industrial (IPI), cuya fuente es el Federal Reserve Bank (FRED, sf), al no encontrar un indicador otro indicador más cercano para esta variable . 
Para el caso del PIB de la cultura y los subsectores que se analizan, la variable más cercana es el mismo PIB, por lo cual se utiliza el método del Filtro de Kalman para convertir la información anual en trimestral, y al mismo IGAE para convertir la información trimestral en mensual, al no encontrar algún otro indicador más cercano para esta parte.

Con respecto a la construcción del Tipo de Cambio Real, esta se realiza de acuerdo con la formulación realizada por Francisco Rivera Bátiz y Luis Rivera Bátiz (op. cit.).

Las partes que se analizan son las exportaciones e importaciones totales del sector de la cultura, así como de los subsectores, a saber:

Las Artes Visuales y Plásticas, están integradas por la producción de Dibujo, Fotografía, Pintura y Escultura, Comercio de Artes Plásticas y Visuales, así como la Gestión Pública en Artes Plásticas y Visuales.

El subsector de Música y Conciertos se conforma por la fabricación de Instrumentos Musicales, la producción en Música, la realización de Conciertos, la Propiedad intelectual en Música, el Comercio de bienes para música, así mismo, lo generado por la Gestión Pública en Música y Conciertos.

El subsector de los Medios Audiovisuales es más complejo, debido a que está integrado por los medios de comunicación como el Cine, el Internet, la Radio, la Televisión, los Videojuegos, así como la Propiedad intelectual en medios audiovisuales, el Comercio en audiovisuales y la Gestión pública en audiovisuales.

\section{Resultados}

De acuerdo con el Banco Mundial, el GAC del total de la economía mexicana durante el periodo de estudio, es superior al 50 por ciento (más no mayor al $80 \%$ ), el cual indica una apertura comercial en crecimiento, al contrario de Estados Unidos que no supera el $30 \%$.

Así, comparando el GAC del total del sector de la cultura que se muestra en la figura 2, se revisa que este es mucho menor que el del total de la economía, lo cual muestra el poco dinamismo que tienen los bienes culturales en el comercio internacional. En la misma figura, se visualiza el comportamiento de los Medios Audiovisuales, el cual es bastante similar y, se puede afirmar, que el comercio cultural es de carácter cerrado. 
Figura 1

Grado de Apertura Comercial (GAC). 2008-2017

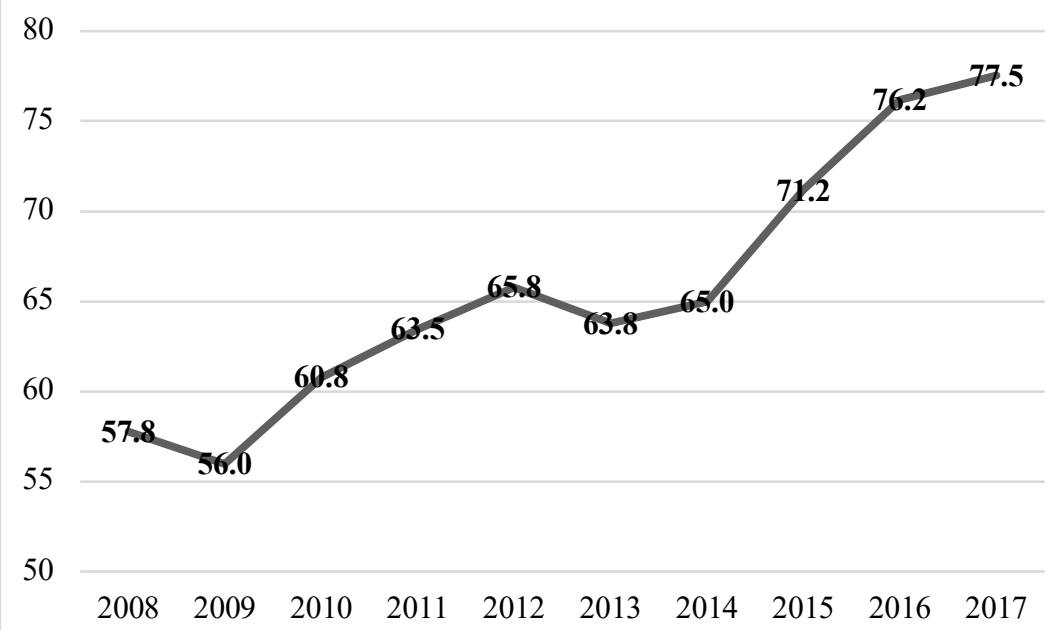

Fuente: Banco Mundial (sf). Indicadores del Desarrollo Mundial.

Figura 2

EI GAC de la Cultura y Medios Audiovisuales

0.25

\begin{tabular}{|lllll|l|l|}
0.20 & $\mathbf{0 . 1 9}$ & & & $\mathbf{0 . 1 9}$ & \\
& & $\mathbf{0 . 1 8}$ & $\mathbf{0 . 1 8}$ & & $\mathbf{0 . 1 7}$
\end{tabular}

0.15

0.10

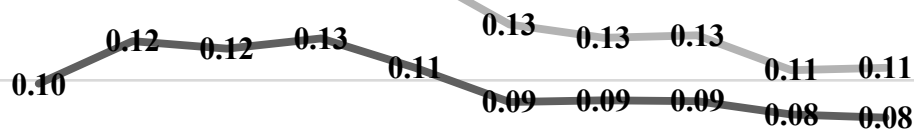

0.05

0.00

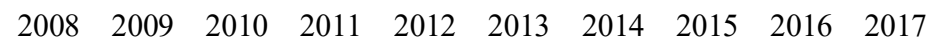

$\longrightarrow$ Total Cultural $\longrightarrow$ Medios Audiovisuales

Fuente: Elaboración Propia a partir del Sistema de Cuenta Satelitales de Cultura. INEGI. 
En la figura 3, se revisa el GAC de las Artes Audiovisuales y de la Música, si bien son demasiado bajos, a diferencia de lo mostrado en la figura 2, se muestra que existe mayor reconocimiento mundial por los creadores y productores de estas áreas, aunque en la segunda variable se pierde el dinamismo en los últimos años.

Por tanto, las condiciones de ser una economía pequeña no genera el dinamismo suficiente para la existencia de una apertura comercial real en lo que respecta a bienes culturales.

Figura 3

GAC Artes Audiovisuales y Música

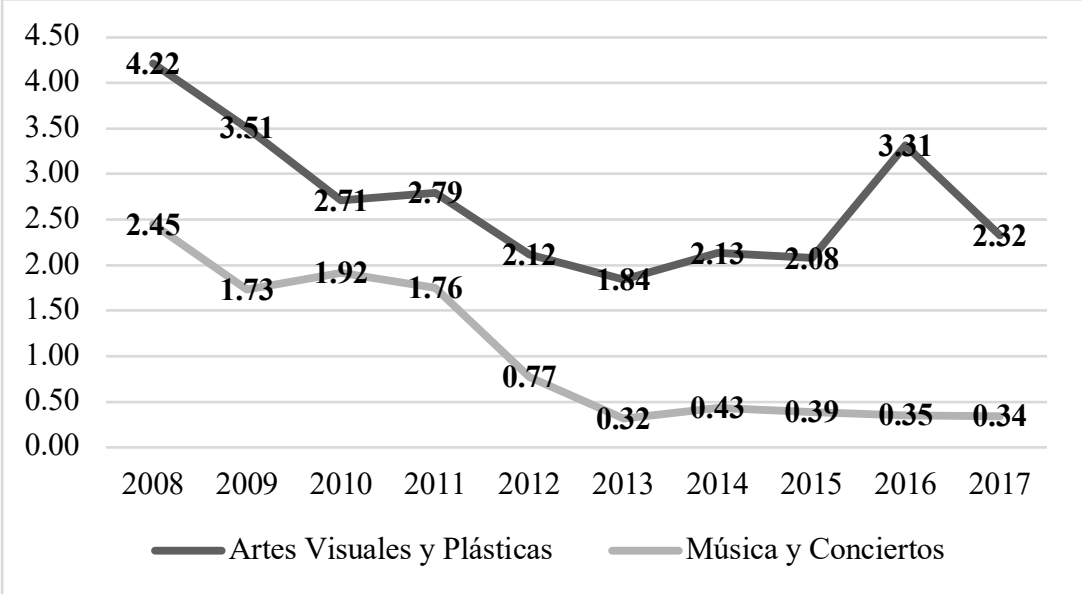

Fuente: Elaboración Propia a partir del Sistema de Cuenta Satelitales de Cultura. INEGI.

Estos resultados de los GAC de la cultura, muestran una tendencia a la baja, además de que, en la mayoría de los casos, no superan el nivel del $1 \%$, durante el periodo de estudio. Por otro lado, la externalidad expresada por Rauch y Trindade (op. cit.), en el sentido de que la cultura es más diversa gracias al comercio, no es visible con estos resultados.

Antes de revisar la llamada Condición Marshall-Lerner, se verifica a través de la Prueba Dickey-Fuller Aumentada (ADF), que todas las variables presentan un nivel de estacionariedad de primer grado. Sin embargo, al aplicar el modelo clásico de regresión lineal, no todas las operaciones resultan estar cointegradas, como se revisa en la tabla 1. 
Tabla 1

Resultados de Pruebas de Cointegración

\begin{tabular}{|c|c|c|c|}
\hline \multirow{2}{*}{$\begin{array}{c}\text { Variable Dependiente } \\
\text { Logaritmo de } \\
\text { Exportaciones totales de } \\
\text { Cultura }\end{array}$} & \multicolumn{2}{|c|}{ Variables Independientes } & \multirow{2}{*}{$\begin{array}{c}\begin{array}{c}\text { Resultado } \\
\text { Cointegración }\end{array} \\
\text { Cointegración* }\end{array}$} \\
\hline & $\begin{array}{l}\text { Logaritmo Tipo } \\
\text { de Cambio Real }\end{array}$ & $\begin{array}{l}\text { Logaritmo } \\
\text { Indice PIB } \\
\text { EEUU }\end{array}$ & \\
\hline $\begin{array}{l}\text { Logaritmo Importaciones } \\
\text { totales de Cultura }\end{array}$ & $\begin{array}{l}\text { Logaritmo Tipo } \\
\text { de Cambio Real }\end{array}$ & $\begin{array}{l}\text { Logaritmo } \\
\text { Indice PIB } \\
\text { México }\end{array}$ & No Cointegración \\
\hline $\begin{array}{l}\text { Logaritmo de } \\
\text { Exportaciones Artes } \\
\text { Visuales y Plásticas }\end{array}$ & $\begin{array}{l}\text { Logaritmo Tipo } \\
\text { de Cambio Real }\end{array}$ & $\begin{array}{l}\text { Logaritmo } \\
\text { Indice PIB } \\
\text { EEUU }\end{array}$ & No Cointegración \\
\hline $\begin{array}{l}\text { Logaritmo de } \\
\text { Exportaciones Artes } \\
\text { Visuales y Plásticas }\end{array}$ & $\begin{array}{l}\text { Logaritmo Tipo } \\
\text { de Cambio Real }\end{array}$ & $\begin{array}{l}\text { Logaritmo } \\
\text { Indice PIB } \\
\text { México }\end{array}$ & Cointegración* \\
\hline $\begin{array}{c}\text { Logaritmo de } \\
\text { Exportaciones Música y } \\
\text { Conciertos }\end{array}$ & $\begin{array}{l}\text { Logaritmo Tipo } \\
\text { de Cambio Real }\end{array}$ & $\begin{array}{l}\text { Logaritmo } \\
\text { Indice PIB } \\
\text { EEUU }\end{array}$ & Cointegración** \\
\hline $\begin{array}{c}\text { Logaritmo de } \\
\text { Importaciones Música y } \\
\text { Conciertos }\end{array}$ & $\begin{array}{l}\text { Logaritmo Tipo } \\
\text { de Cambio Real }\end{array}$ & $\begin{array}{l}\text { Logaritmo } \\
\text { Indice PIB } \\
\text { México }\end{array}$ & No Cointegración \\
\hline $\begin{array}{l}\text { Logaritmo de } \\
\text { Exportaciones Medios } \\
\text { Audiovisuakes }\end{array}$ & $\begin{array}{l}\text { Logaritmo Tipo } \\
\text { de Cambio Real }\end{array}$ & $\begin{array}{l}\text { Logaritmo } \\
\text { Indice PIB } \\
\text { EEUU }\end{array}$ & No Cointegración \\
\hline $\begin{array}{c}\text { Logaritmo de } \\
\text { Importaciones Medios } \\
\text { Audiovisuakes }\end{array}$ & $\begin{array}{l}\text { Logaritmo Tipo } \\
\text { de Cambio Real }\end{array}$ & $\begin{array}{l}\text { Logaritmo } \\
\text { Indice PIB } \\
\text { México }\end{array}$ & Cointegración* \\
\hline
\end{tabular}

Por tal motivo, se confirma que el desarrollo de modelos vectoriales autorregresivos (VAR) o de Corrección de Error (VEC) no son los adecuados, debido a que se requiere de una uniformidad de resultados que sea confiable. Además, todos los residuos muestran evidencia de presencia de heterocedasticidad, de ahí la importancia de contemplar modelos como ARCH-GARCH.

Considerando el modelo expuesto en (4) y (4a), se analizan cada una de las partes del comercio de bienes culturales. Los resultados que corresponden a los Medios Audiovisuales se muestran en la tabla 2. 
Tabla 2

Resultados de Balanza Comercial de Medios Audiovisuales

\begin{tabular}{|c|c|c|}
\hline & $\begin{array}{r}\text { Diferencial del } \\
\text { de } \mathrm{M}\end{array}$ & $\begin{array}{l}\text { de Exportaciones } \\
\text { visuales }\end{array}$ \\
\hline \multirow{3}{*}{$\begin{array}{l}\text { Diferencial del Logaritmo del Tipo } \\
\text { de Cambio Real }\end{array}$} & $-0.1506^{* *}$ & $-0.0024 * *$ \\
\hline & $(0.0753)$ & $(0.0012)$ \\
\hline & {$[-2.00]$} & {$[-2.01]$} \\
\hline \multirow{3}{*}{$\begin{array}{l}\text { Diferencial del Logaritmo del } \\
\text { Índice del PIB Estados Unidos }\end{array}$} & $1.3755^{*}$ & \\
\hline & $(0.2213)$ & \\
\hline & {$[6.22]$} & \\
\hline \multirow{3}{*}{$\begin{array}{l}\text { Diferencial del Logaritmo del } \\
\text { Índice del PIB México }\end{array}$} & & $1.0020 *$ \\
\hline & & $(0.2213)$ \\
\hline & & {$[6.22]$} \\
\hline \multirow{3}{*}{ Constante } & & $-0.0001 * * *$ \\
\hline & & $(0.0001)$ \\
\hline & & {$[-1.86]$} \\
\hline \multirow{3}{*}{$\operatorname{AR}(6)$} & $-0.1485^{*}$ & $0.5029 *$ \\
\hline & $(0.0531)$ & $(0.0258)$ \\
\hline & {$[-2.80]$} & [19.53] \\
\hline \multirow{3}{*}{$\mathrm{AR}(12)$} & & $0.5029 *$ \\
\hline & & $(0.0258)$ \\
\hline & & [19.53] \\
\hline \multirow{3}{*}{ MA(3) } & & $-0.0191 *$ \\
\hline & & $(0.0054)$ \\
\hline & & {$[-3.54]$} \\
\hline \multirow{3}{*}{ MA(6) } & & $-0.0607 *$ \\
\hline & & $(0.0039)$ \\
\hline & & {$[-15.49]$} \\
\hline \multirow{3}{*}{$\mathrm{ARCH}(12)$} & $1.3743^{*}$ & $3.6182 *$ \\
\hline & $(0.3615)$ & $(0.3242)$ \\
\hline & {$[3.80]$} & [11.16] \\
\hline \multirow{3}{*}{ GARCH(12) } & $0.2497 * *$ & \\
\hline & $(0.1166)$ & \\
\hline & {$[2.14]$} & \\
\hline
\end{tabular}

Fuente: Elaboración propia. Nota: () Error Estándar, OMG. [], Estadístico z. ${ }^{*}$ Nivel de Significancia del 0.01, ** Nivel de Significancia del 0.05, *** Nivel de Sigficancia del 0.10.

Este resultado es relevante pues, tanto exportaciones como importaciones, resultan negativos ante una depreciación del tipo de cambio real, esto significa que la balanza comercial de los medios audiovisuales empeora tanto por su oferta como por su demanda. Otra lectura señala que las relaciones comerciales, en este rubro, son demasiado inelásticas.

En la tabla 3, se muestran los resultados sobre el comercio en Música y Conciertos. Si bien son parecidos a los mostrados en la tabla 2, se presenta que, en términos del ingreso estadounidense, las exportaciones tienen un comportamiento inelástico, mientras que las importaciones son cercanas a la 
elasticidad unitaria con respecto a los ingresos domésticos. También se puede afirmar que la depreciación encarece su comercio, con el exterior.

Tabla 3

Resultados de la Balanza Comercial en Música y Conciertos

Diferencial del Logaritmo de Exportaciones de Música y Conciertos

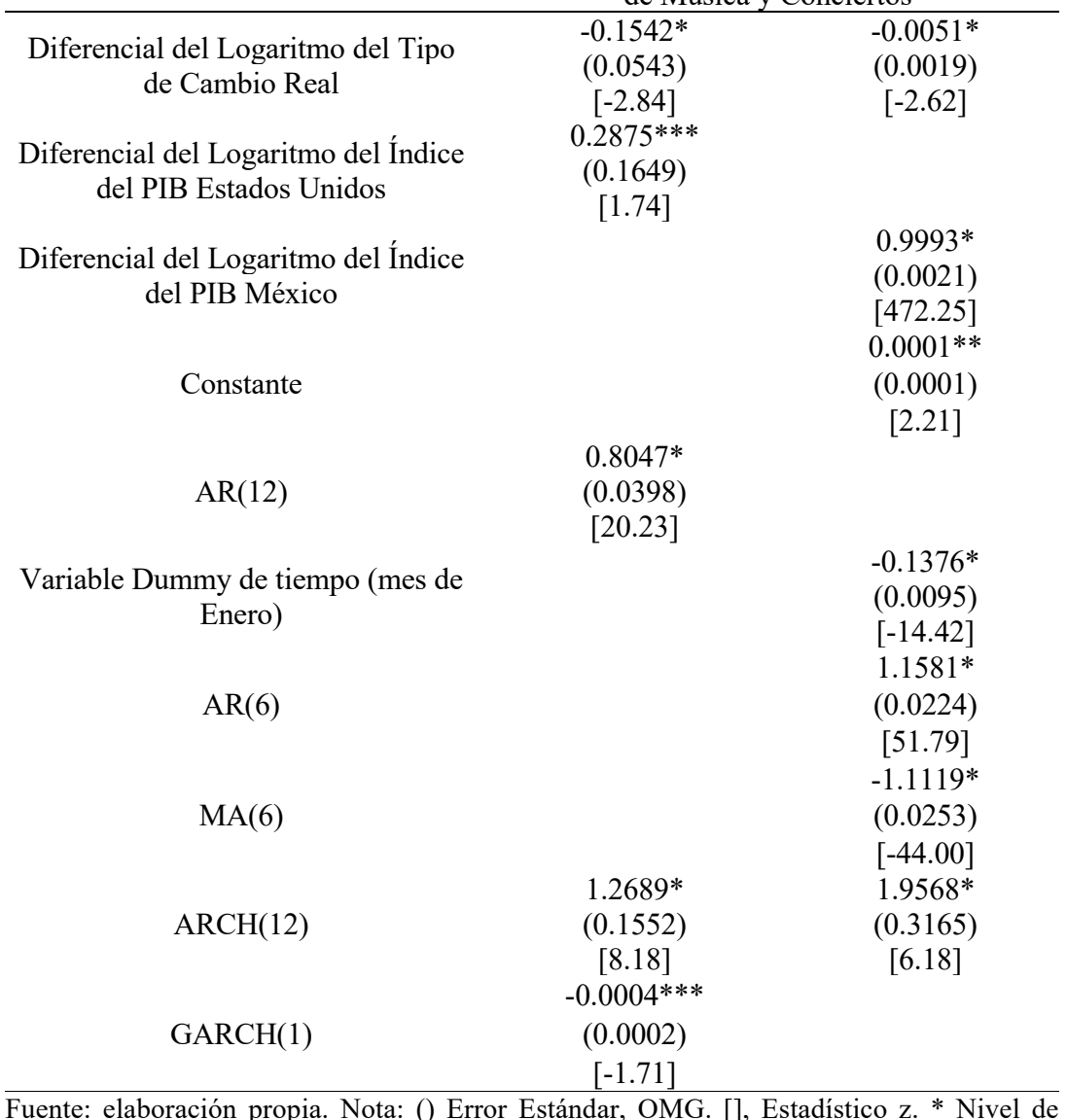
Significancia del 0.01, ** Nivel de Significancia del $0.05,{ }^{* * *}$ Nivel de Sigficancia del 0.10.

Con respecto a las Artes Visuales y Plásticas (tabla 4), el comportamiento es muy similar a la de los subsectores anteriores. Esto implica que las exposiciones de los artistas plásticos en el exterior no superan a las de los artistas de otros países, puesto que los resultados señalan que la depreciación encarece este tipo de actividades en el exterior. 


\section{Tabla 4}

Resultados de la Balanza Comercial en Artes Visuales y Plásticas

\begin{tabular}{|c|c|c|}
\hline & $\begin{array}{r}\text { Diferencial del Lo } \\
\text { Artes V }\end{array}$ & $\begin{array}{l}\text { xportaciones de } \\
\text { ticas }\end{array}$ \\
\hline \multirow{3}{*}{$\begin{array}{l}\text { Diferencial del Logaritmo del Tipo } \\
\text { de Cambio Real }\end{array}$} & $-0.1462 *$ & $-0.0026^{*}$ \\
\hline & $(0.0535)$ & $(0.0013)$ \\
\hline & {$[-2.73]$} & {$[-2.10]$} \\
\hline \multirow{3}{*}{$\begin{array}{c}\text { Diferencial del Logaritmo del } \\
\text { Índice del PIB Estados Unidos }\end{array}$} & $0.3068 * * *$ & \\
\hline & $(0.1602)$ & \\
\hline & [1.92] & \\
\hline \multirow{3}{*}{$\begin{array}{l}\text { Diferencial del Logaritmo del } \\
\text { Índice del PIB México }\end{array}$} & & $0.9983^{*}$ \\
\hline & & $(0.0013)$ \\
\hline & & {$[770.23]$} \\
\hline \multirow{3}{*}{ Constante } & $-0.0185 * * *$ & \\
\hline & $(0.0095)$ & \\
\hline & {$[-1.95]$} & \\
\hline \multirow{3}{*}{$\operatorname{AR}(12)$} & $0.8652 *$ & \\
\hline & $(0.0214)$ & \\
\hline & [40.36] & \\
\hline \multirow{3}{*}{$\operatorname{AR}(3)$} & & $-0.0185^{*}$ \\
\hline & & $(0.0047)$ \\
\hline & & {$[-3.95]$} \\
\hline \multirow{3}{*}{$\operatorname{AR}(6)$} & & $0.0430 *$ \\
\hline & & $(0.0160)$ \\
\hline & & [2.69] \\
\hline \multirow{3}{*}{ MA(12) } & & $0.3180 *$ \\
\hline & & $(0.0331)$ \\
\hline & & {$[9.60]$} \\
\hline \multirow{3}{*}{ ARCH(12) } & $1.4581^{*}$ & $2.1134 *$ \\
\hline & $(0.2432)$ & $(0.2533)$ \\
\hline & {$[6.00]$} & [8.34] \\
\hline
\end{tabular}

Fuente: elaboración propia. Nota: () Error Estándar, OMG. [], Estadístico z. ${ }^{*}$ Nivel de Significancia del $0.01, * *$ Nivel de Significancia del 0.05 , *** Nivel de Significancia del 0.10 .

Al analizar la Balanza Comercial del sector de la cultura (tabla 5), se muestran resultados similares a los de cada subsector, lo cual indica que su dinámica comercial es más lenta en comparación con el resto de la economía.

Aunado a lo expuesto en las figuras 2 y 3, la apertura comercial no ha beneficiado al sector de la cultura, convirtiendo a México en un receptor de las actividades culturales del exterior. 
Tabla 5

Balanza Comercial del Total del Sector de la Cultura

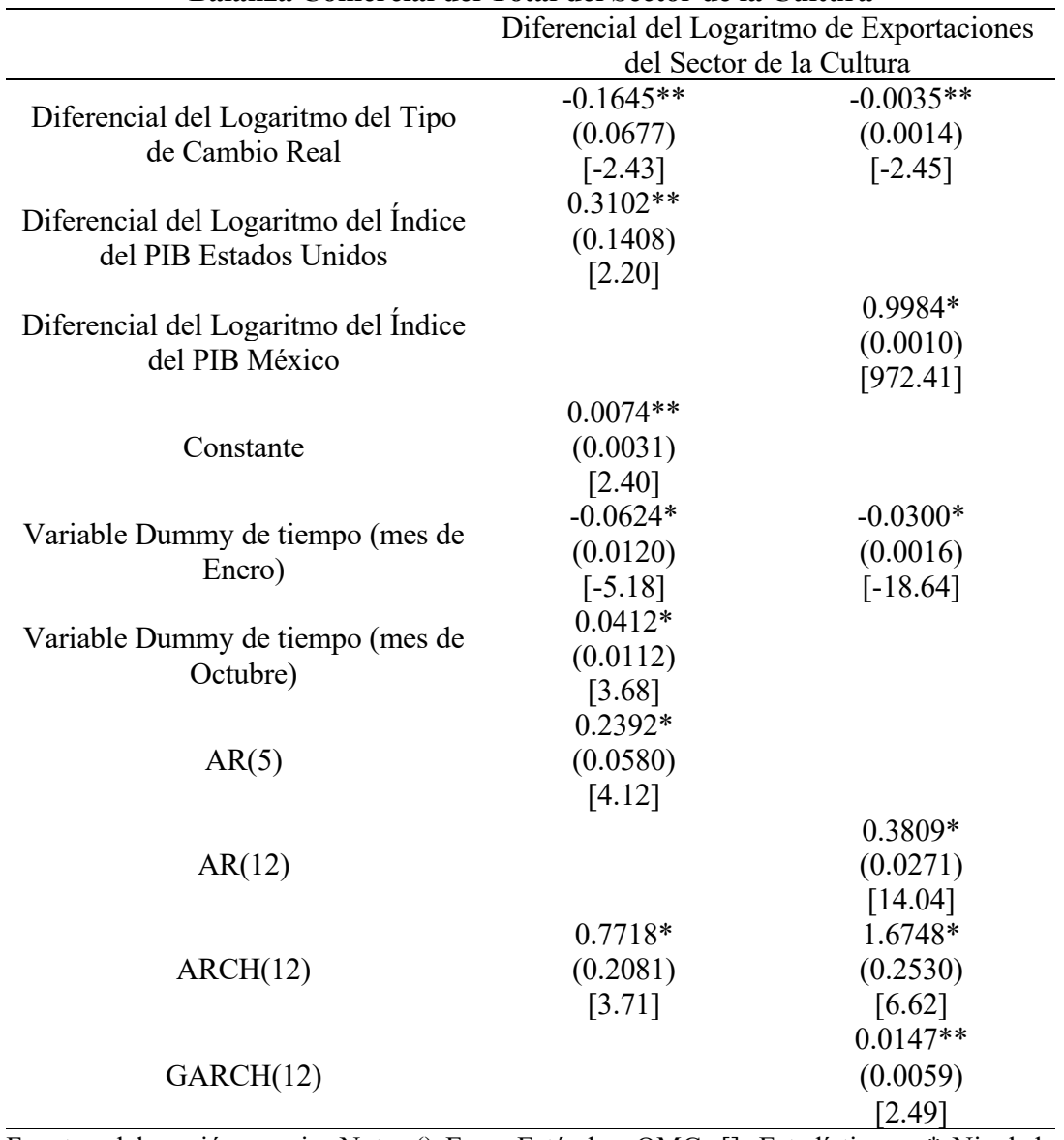

Fuente: elaboración propia. Nota: () Error Estándar, OMG. [], Estadístico z. ${ }^{*}$ Nivel de Significancia del 0.01, ** Nivel de Significancia del 0.05, *** Nivel de Significancia del 0.10 .

Sobre la accesibilidad del consumo, con excepción de los medios audiovisuales, se observa un comportamiento inelástico frente al ingreso estadounidense, lo cual muestra un interés demasiado bajo por el consumo de bienes culturales de México.

Para el caso de las elasticidades de las importaciones, con respecto a los ingresos domésticos, se muestra una elasticidad unitaria, o al menos cerca de la unidad. Esto implica que el consumo en bienes culturales, provenientes del exterior, depende de los ingresos domésticos por encima de los efectos negativos que pueda contraer una depreciación de la moneda nacional. 
Como afirman Rauch y Trindade (op. cit.), estos resultados no muestran una red de externalidades de consumo en cultura, al contrario, se da la razón a Tania Voon (op. cit.), debido a la evidencia de que hay un dominio en bienes culturales provenientes de las economías grandes. Lo cual parece mostrar que, los consumidores de los productos extranjeros son susceptibles a la confianza sobre estos bienes y servicios culturales, tal y como afirma Einarsson (op. cit.). Por último, en la tabla 6, se establece la operación Marshall-Lerner, en la que todos los resultados muestran ser menores de la unidad.

\section{Tabla 6}

Resultados Condición Marshall-Lerner

\begin{tabular}{lccc}
\hline & $\begin{array}{c}\text { Elasticidad-Precio } \\
\text { Exportaciones (Valor } \\
\text { Absoluto) }\end{array}$ & $\begin{array}{c}\text { Elasticidad-Precio } \\
\text { Importaciones (Valor } \\
\text { Absoluto) }\end{array}$ & $\begin{array}{c}\text { Condición } \\
\text { Marshall- } \\
\text { Lerner }\end{array}$ \\
\hline $\begin{array}{l}\text { Total Cultura } \\
\text { Artes Plásticas y }\end{array}$ & 0.1645 & 0.0035 & 0.1680 \\
$\begin{array}{l}\text { Visuales } \\
\text { Música y }\end{array}$ & 0.1462 & 0.0026 & 0.1488 \\
$\begin{array}{l}\text { Conciertos } \\
\begin{array}{l}\text { Medios } \\
\text { Audiovisuales }\end{array}\end{array}$ & 0.1542 & 0.0051 & 0.1593 \\
\hline
\end{tabular}

Fuente: elaboración Propia a partir de los resultados anteriores.

Los resultados implican que un efecto de depreciación de la moneda nacional, no mejora las condiciones de la balanza comercial del sector de la cultura, mostrando que es demasiado dependiente del exterior.

La influencia de mercado de una economía grande, como la de Estados Unidos frente a México, representa el tamaño de un mercado cultural desigual a favor del vecino del norte, puesto que esta competencia de bienes no solo es de mercado sino también de tecnologías como bien ya lo menciona Kónya (op. cit.).

Al aplicar el modelo para el análisis de la Condición Marshall-Lerner, siguiendo la propuesta de regresión realizada por Baltazar y Ramos (op. cit.), los resultados de cultura no superan el orden del 0.168 , correspondiente al total de este sector. Por consiguiente, no hay una mejora en la Balanza Comercial de la cultura, por lo que una depreciación encarece las actividades creativas, de acuerdo con lo mostrado en la última tabla.

Por tanto, el tamaño de la economía influye en el comercio de bienes culturales pues, ante la depreciación, se encarece la producción interna, y aunque el efecto también es negativo en las importaciones, parece ser más accesible el producto exterior en el mercado interno. 


\section{Conclusiones}

Este trabajo representa un ejercicio de comercio internacional sobre bienes culturales, lo cual implica un análisis propio de la economía mexicana frente al mercado exterior, principalmente, el estadounidense, el principal socio comercial de nuestro país.

Los resultados muestran que, ante la presencia de una economía grande como la de Estados Unidos, México no ha mostrado la capacidad de competencia en lo referente a bienes culturales, a pesar de tener una alta presencia migrante en el país vecino del Norte.

Con respecto a las condiciones de consumo de bienes culturales, estos dependen más de los ingresos que del precio determinado por el tipo de cambio real. Esto ocurre más para el caso de los productos importados, pero no ocurre para las exportaciones, con excepción de los medios audiovisuales, debido a las condiciones tecnológicas de la digitalización y las redes sociales.

Se observa que existe una alta influencia en las actividades de consumo doméstico por los bienes culturales del exterior, debido a la confianza que las mismas empresas "creativas" extranjeras generan, a pesar de existir productos similares, de carácter nacional, con cierto reconocimiento internacional.

Los resultados propios de la Condición Marshall-Lerner, para el sector de la cultura, sugieren que ante una depreciación empeoran las circunstancias propias de su balanza comercial, al contrario de lo que ocurre en el total de la economía nacional. Parece que para nuestro país existe una reducción de la diversidad cultural en términos del comercio de los bienes, de acuerdo con la teoría expuesta por Bisin y Verdier (op. cit.).

Si bien en nuestro país existen una gran cantidad de artistas, bailarines, pintores, escultores, cineastas y literatos, reconocidos en el nivel internacional, los resultados obtenidos muestran que el grado de influencia cultural de México, no es demasiado grande en comparación con los bienes culturales provenientes del exterior.

Aunado a lo último, los resultados del modelo ARCH-GARCH, para la Condición Marshall-Lerner, reafirman lo dicho en la hipótesis: que a pesar de la existencia de artistas nacionales con proyección mundial, la influencia cultural de México es mínima. Las razones están sustentadas por el tamaño de la economía, como las preferencias de consumo de bienes culturales provenientes del exterior 
Einarsson (op. cit.) afirma que muchos de los creadores culturales trabajan a través de agentes o agencias para que les sea posible ejercer su oficio en el exterior. Sin embargo, en la mayoría de los casos, sus actividades resultan ser autogestivas, tal como parecen indicar los resultados de los artistas plásticos y de los músicos, pues ante una depreciación, su actividad se encarece. Esto señala que la depreciación real afecta a las actividades artísticas, encareciendo la labor de trabajadores independientes y empresas culturales.

\section{Referencias}

[1] Baltazar, J., Ramos, M. (2014), México: Comercio Exterior, Producto y Condición de Marshall-Lerner. En Tiempo Económico. Vol. IX No. 27, pp. 5-26. México. Universidad Autónoma Metropolitana-Azcapotzalco.

[2] BEA, (sf), Data by Topic. Consultado el 12 de marzo de 2019. Recuperado de https://www.bea.gov/data

[3] Bisin, A., Verdier, T., (2014), Trade and Cultural Diversity. En Handbook of the Economics of Art and Culture, Ginsburgh, V. \& Throsby D. (Edit.), pp. 440483. Reino Unido. North Holand-Elsevier.

[4] Bustamante, R., Morales, F. (2009), Probando la Condición de Marshall-Lerner y el Efecto Curva-J: Evidencia Empírica para el Caso Peruano. En Estudios Económicos, No. 16, pp. 103-126, Marzo. Lima, Perú. Banco Central de Reserva del Perú. Consultado el 24 de febrero de 2019. Recuperado de http://www.bcrp.gob.pe

[5] CAAV, (sf), La historia de la Animación en México. Parte 1. Consultado el 2 de abril de 2019. Recuperado de https://caav.mx/la-historia-de-la-animacion-enmexico-parte-1/

[6] Einarsson, Á. (2016), Cultural Economics. Iceland. Bifröst University Press.

[7] Elizondo, R. (2012), Estimaciones del PIB Mensual Basadas en el IGAE. En Documentos de Investigación del Banco de México. No. 2012-11. Banco de México. Consultado el 5 de febrero de 2019. Recuperado de http://www.banxico.org.mx

[8] González, E. (2013), Memorias de la Censura o Uruchurtu, el Regente de Hierro. Consultado el 21 de abril de 2019. Recuperado de https://www.contralinea.com.mx/

[9] INEGI, (sf), Banco de Información Económica. Consultado el 2 de diciembre de 2018. Recuperado de https://www.inegi.org.mx/

[10] , (2018), Cuenta Satélite de la Cultura de México, 2017. Consultado el 4 de febrero de 2019. Recuperado de https://www.inegi.org.mx

[11] Kónya, I. (2006), Modeling Cultural Barriers in International Trade. En Review of International Economics. Vol. 14 No. 3, pp. 494-507. John Wiley and Son Ed. EE.UU.

[12] López, R. (2014), Medición del Grado de Apertura de una Economía. En Revista eXtoicos. No. 14, pp. 87-88.

[13] OCDE (2010), Measuring Globalisation. Economic Globalisation Indicators. Paris, OECD Publishing. Consultado el 28 de abril de 2019. Recuperado de https://www.oecd-ilibrary.org 
[14] Ramírez, A., Azuara, A., Calderón, M., Candelaria, L. (2008), Condición MarshallLerner: una aplicación a México. Tiempo Económico, Vol. III No. 8, pp. 4157. México. Universidad Autónoma Metropolitana-Azcapotzalco.

[15] Rauch, J., Trindade, V. (2009), Neckties in the Tropics: a Model of International Trade and Cultural Diversity. Canadian Journal of Economics, Vol.42, No. 3, Canada, pp. 809-843. Canada Economics Association.

[16] Rivera-Batiz, F., Rivera-Batiz, L. A. (1993), International Finance and Open Economy Macroeconomics. Columbia University, USA. 2nd Revised Edition. Pearson Edit.

[17] Voon, T. (2007), Cultural Products and the World Trade Organization. Legal Studies Reserch Paper, No. 342, pp. 1-35. Cambridge University Press. Consultado el 30 de abril de 2019. Recuperado de https://papers.ssrn.com 